\title{
URSULA KLEINHENZ
}

\section{Zur Typologie phonologischer Domänen*}

\begin{abstract}
Eine aktuelle Debatte in der Phonologie betrift den Status phonologischer Konstituenten oberhalb des Wortes, insbesondere von kleineren phonologischen Einheiten innerhalb von Intonationsphrasen. In vielen Sprachen haben solche Phrasengrenzen eine phonologische Funktion. Offenbar bedienen sich andere Sprachen dieser Möglichkeit aber nicht.

Ich möchte hier zwei Fragen diskutieren: Erstens, ob man für die Sprachen der zweiten Gruppe annehmen soll, daB sie ebenfalls über diese Kategorie verfügen, diese also abstrakt in ihrem System vorhanden ist, und zweitens, welche Einsichten wir aus der Gruppe von Sprachen, in denen die phonologischen Phrase eine Funktion hat, gewinnen kōnnen. Besteht irgendeine Korrelation zwischen dieser Eigenschaft von Sprachen und anderen phonologischen Eigenschaften dieser auf den ersten Blick inkonsistenten Gruppe?

Ich argumentiere, daß es nicht sinnvoll ist $\mathrm{zu}$ behaupten, daß die phonologische Phrase eine universelle Kategorie ist, weil man dann eine typologische Generalisierung über Sprachen verlieren würde. Ich zeige in diesem Beitrag, daß die Tendenz von Sprachen, Phrasengrenzen eine phonologische Funktion zuzuweisen, mit rhythmischen Eigenschaften auf der Wortebene korreliert. Anschließend mache ich einen Vorschlag zur Revision der phonologischen Kriterien der Typologie des Sprachrhythmus.
\end{abstract}

\section{Die Funktion von Intonationseinheiten}

Alle Sprachen phrasieren, d.h., stets werden Sätze in der gesprochenen Sprache in kleinere Intonationseinheiten eingeteilt. Diese phonetische Phrasierung ist universell; Sprachen unterscheiden sich lediglich in der Art der akustischen Realisierung dieser Phrasierung (Pitch-Akzent, Längung etc.). Die Rolle dieser Phrasen in der Phonologie ist jedoch umstritten.

Generell müssen Konstituenten bestimmte Kriterien erfüllen, bevor man annimmt, daß sie eine phonologische „Funktion” haben. Man vergleiche die folgende Liste (1).

* Für Hinweise und Kommentare danke ich T.A. Hall, Ewald Lang, Sylvia Löhken, Christine Mooshammer und Iggy Roca. 
(1) Kriterien für phonologische Konstituenten (vgl. Selkirk 1980; Rice 1990)

Eine Konstituente muß eine Domäne eigener Art sein, d.h., sie darf nicht isomorph mit anderen Domänen sein (z.B. dürfen Phonologische Phrasen nicht immer identisch mit einer syntaktischen Phrase sein).

Sie muB aber (mit ihren eigenen Regeln) abgeleitet werden können: es darf keine zufällige oder willkürliche Domäne sein.

Regeln müssen sich systematisch auf sie beziehen, d.h. entweder ist die Konstituente selber die Domäne einer Regel oder die Grenzen der Konstituente blockieren eine Regel. Auf diese Weise kann man die Grenzen der Domäne nachweisen.

In vielen Sprachen korrelieren phonologische Domänen nicht mit den vergleichbaren syntaktischen Einheiten (z.B. Wort, Phrase). Die prosodische Hierarchie in (2) zeigt alle Konstituenten, die in der Phonologie eine Funktion haben können.

(2) Prosodische Hierarchie (vgl. Nespor/Vogel 1986)

Äußerung

Intonationsphrase

Phonologische Phrase

Klitikgruppe

Phonologisches Wort

FuB

Silbe

subsilbische Konstituenten

\subsection{Phonologische Phrasen}

Wie gesagt sind nicht alle diese Einheiten unumstritten. Während Phonologen z.B. implizit oder explizit davon ausgehen, da B es Intonationsphrasen oder Silben gibt, herrscht Uneinigkeit über die Rolle der Phonologischen Phrase. Die Phonologische Phrase hat offenbar nicht in allen Sprachen eine Funktion. Frota (1995) 2.B. hat alle in Frage kommenden Regeln des Portugiesischen untersucht und festgestellt, daB keine davon auf Domänen vom Format der phonologischen Phrase operiert.

Diese Feststellung allein läBt noch keine Aussage über die Relevanz der phonologischen Phrase zu. Zwar haben manche Autoren vorgeschlagen, $\mathrm{da} B$ nicht alle prosodischen Konstituenten universell sind (z.B. Auer 1994 für die Silbe), aber solange davon keine weiteren Argumente abhängen, 
könnte man ebensogut (oder besser) davon ausgehen, daB die prosodische Hierarchie universell ist, man aber in einigen Sprachen die Grenzen bestimmter Konstituenten in der Phonologie nicht sehen kann. Bevor ich näher auf diese Argumente eingehe, erläutere ich kurz, was man genau unter der Kategorie „phonologische Phrase” versteht.

Die phonologische Phrase ist ursprünglich in der einschlägigen Literatur eine rhythmische Einheit. Sie wurde zunächst nur verwendet, um rhythmisch bedingte Akzentverschiebungen zu beschreiben. Bekannte Beispiele habe ich in (3) aufgelistet. Hauptakzente sind mit Großbuchstaben markiert, Nebenakzente mit ' . Von der syntaktischen Struktur her handelt es sich bei den Beispielen in (3) jeweils um Nominalphrasen.

(3) Akzentverschiebung innerhalb „phonologischer Phrasen” (vgl. Giegerich 1985)
(a) thirTEEN
aber
thírteen BAbies
(b) PaderBORN
aber
Páderborner UNi
(c)
aber
die UNi von Pader BORN

Nur in (3 c) wird keiner der Hauptakzente reduziert. Als Grund hierfür wird häufig genannt, daß es eine rhythmische Einheit, eben die phonologische Phrase, gibt, innerhalb derer es nur einen Hauptakzent geben kann. Stoßen zwei Hauptakzente innerhalb einer phonologischen Phrase zusammen, so wird einer davon reduziert („Rhythm Rule”). [Paderborner $U n$ i] wäre in diesem Beispiel eine einzige Phrase, während [die Uni] [von Paderborn] aus zwei Phrasen bestünde. Der Akzent kann demnach in (3 c) auf Paderborn bleiben, weil zwischen den beiden Akzenten eine phonologische Phrasengrenze liegt, die die Rhythm Rule blockiert.

Phonologische Phrasen werden durch einen Algorithmus, den ich in (4) zitiert habe, abgeleitet. ${ }^{1}$

(4) (a) Ableitung phonologischer Phrasen

(Nespor/Vogel 1986, S. 168)

The domain of $\mathrm{PhP}$ consists of a CG [=Clitic Group] which contains a lexical head $(\mathrm{X})$ and all CGs on its nonrecursive side up to the CG that contains another [lexical] head outside the maximal projection of $\mathrm{X}$.

(b) Restrukturierung phonologischer Phrasen

(Nespor/Vogel 1986, S. 173)

1 Die in der Ableitung verwendete Kategorie der "Klitikgruppe” ist hier nicht von Bedeutung. Man kann stattdessen auch „Wort” einsetzen. 
A nonbranching $\mathrm{PhP}$ which is the first complement [or adjunct] of $\mathrm{X}$ on its recursive side is joined into the $\mathrm{PhP}$ that contains $\mathrm{X}$.

Phonologische Phrasen werden also im Prinzip aus maximalen syntaktischen Projektionen abgeleitet. In einer Zwischenstufe entsprechen sie den syntaktischen Phrasen. Durch die „Restrukturierung” werden die eigentlichen phonologischen Phrasen erzeugt. Dabei fallen die intermediären Phrasen teilweise zusammen. Die Restriktion hierüber betrifft die Länge der Konstituenten, also kein syntaktisches Kriterium.

Dies wird in (5) anhand des Italienischen demonstriert ( $\phi$ ist die Abkürzung für "phonologische Phrase").

(5) Anwendung von (4) auf das Italienische (vgl. Nespor/Vogel 1986, S. 171)
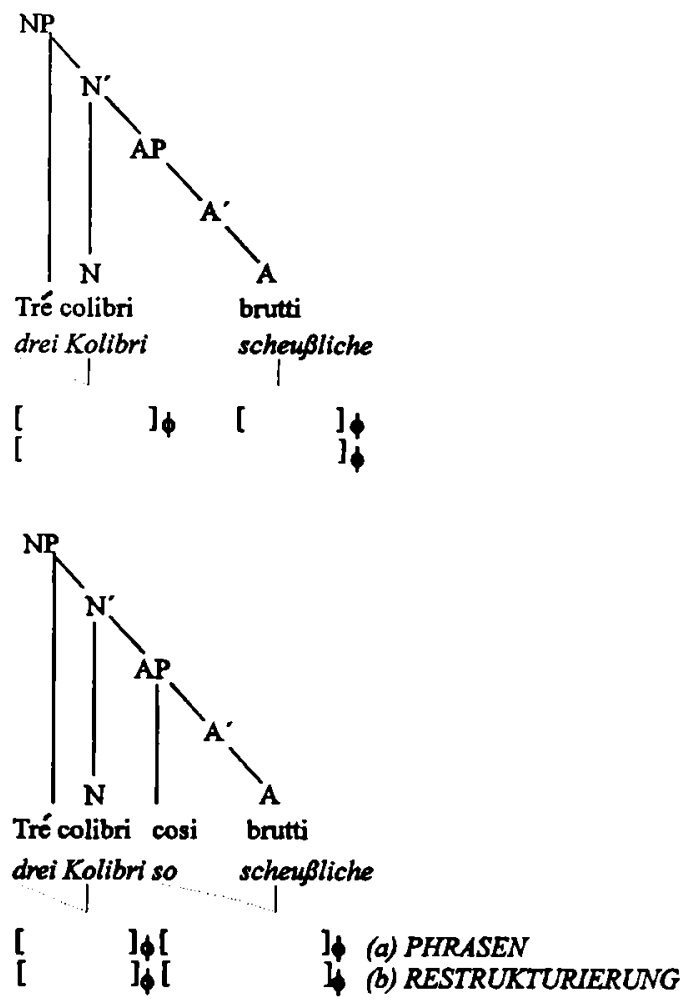
Zuerst werden vom am weitesten rechts stehenden lexikalischen Kopf aus nach links (da das Italienische eine rechtsverzweigende Sprache ist) Phrasen konstruiert. Anschließend werden diese "vorläufigen" Phrasen in die vorangehende Phrase inkorporiert, sofern sie nicht verzweigen. Diese Restriktion verhindert die Bildung überlanger Phrasen.

Die phonologische Phrase im Italienischen ist phonologisch relevant, d.h., sie entspricht den in (1) zusammengefaBten Kriterien. Es gibt z.B. eine segmentale Regel, das radoppiamento sintattico (kurz RS), die Konsonanten längt (Nespor/Vogel 1986, S. 38). RS längt wortinitiale Konsonanten, wenn bestimmte Bedingungen erfüllt sind. Diese Bedingungen sind regional unterschiedlich und spielen im vorliegenden Zusammenhang keine Rolle. Hier ist lediglich zu beachten, daB ein wortinitialer Konsonant nur dann gelängt wird, wenn das vorangehende Wort auf einen betonten Vokal auslautet und die beiden Wörter in derselben phonologischen Phrase sind. Aus (6) bis (8) wird deutlich, daB tatsächlich die phonologische, und nicht die syntaktische Phrasierung ausschlaggebend ist.

(6) Radoppiamento Sintattico (RS) im Italienischen (Region Toskana)

$\begin{array}{llllll}\text { (a) Ho } & \text { visto } & \text { tré } & {[\mathrm{k}: \text { ]olibri }} & \text { [b:]rutti } \\ & \text { Ich } & \text { habe gesehen } & \text { drei } & \text { Kolibri } & \text { scheußliche } \\ \text { (b) Ho } & \text { visto } & \text { tré } & {[\mathrm{k}: \text { ]olibri }} & \text { [k]osi } & \text { [b:]rutti } \\ & \text { Ich } & \text { habe gesehen } & \text { drei Kolibri so } & \text { scheußliche }\end{array}$

Wichtig ist der fettgedruckte Konsonant nach colibri. Er wird in (6b) nicht gelängt, obwohl die segmentale Umgebung (wortinitial, nach einem betonten Vokal) gegeben wäre. Auch die syntaktische Beziehung zwischen colibri/brutti bzw. colibri/cosi ist dieselbe, wie in (7) dargestellt.

(7) Syntaktische Phrasierung von (6)

(Nespor/Vogel 1986, S. 40)
a. [tré
b. [tré [colibri
[colibrí
$\left[\begin{array}{ll} & {[\text { brutti]]] }} \\ {[\text { cosi }} & \text { [brutti]]] }\end{array}\right.$

Relevant für die Anwendung der Regel ist nicht der syntaktische Kontext der $\ddot{A} u ß e r u n g$, sondern ihre phonologische Phrasierung. Diese hängt mit der einfachen Länge der Äußerung zusammen, wie man in (8) erkennen kann: durch die Hinzufügung von cosi in $(8 b)$ entsteht eine zweite verzweigende Phrase. Die beiden Phrasen können nun nicht mehr zusammenfallen. 
(8) Phonologische Phrasierung von (6)
a. [tré colibrí
brutti $]_{\phi}$
b. [tré colibri $]_{\phi}[\operatorname{cosi} \text { brutti }]_{\phi}$

RS betrifft also Konstituenten, die syntaktisch gesehen keine sind. Wenn man z.B. sagen würde, daB RS in der syntaktischen Domäne NP angewandt wird, wäre cosi in (8b) genau so betroffen wie brutti.

Aber nicht in allen Sprachen findet man so deutliche Evidenz für phonologische Phrasen als Kategorie in der Phonologie.

Ein solcher Fall ist das Deutsche. Wiese (1995) argumentiert $\mathrm{zwar}$, daß es phonologische Phrasen (PPh) im Deutschen gibt (wiedergegeben in (9)).

(9) Phonologische Phrasen im Deutschen (vgl. Wiese 1995, S. 71)

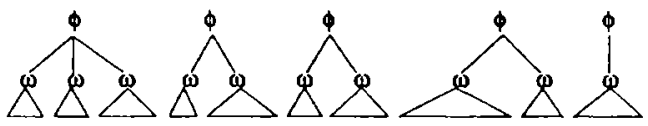

Phonologische Phrasen

Phonologische Wörter

Der alte Mann am Strand fing heute besonders viele Fische

Diese Repräsentation entspricht aber nicht dem Algorithmus von Nespor und Vogel, den ich in (4) wiedergegeben habe, da dort Präpositionalphrasen wie [am Strand] keine eigene phonologische Phrase bilden (im Modell von Nespor/Vogel lösen nur die lexikalischen Köpfe ${ }^{2}$ N, V, A, aber nicht $P$ die Bildung einer phonologischen Phrase aus). Intuitiv sind für den Satz in (9) auch andere Phrasierungen denkbar, vor allem die in $(10)$, in welcher die PP keine eigene Phrase ist: ${ }^{3}$

2 Nespor und Vogel nehmen an, daß nur $N, V$ und $A$ lexikalische Kōpfe sind. Für Leser, die diesem Argument nicht folgen, schlagen sie eine Revision ihres Algorithmus vor, der nur N, V und A-Kategorien einbezieht (Nespor/Vogel 1986, S. 169).

3 Wiese muß aus unabhāngigen Grūnden annehmen, daß der Algorithmus von Nespor/Vogel auch PPs einschließt: Als Evidenz für phonologische Phrasen verwendet er eine Regel, die Koordinationsreduktion, die aber auch für PPs gilt. Diese Regel kann hier nicht besprochen werden (vgl. aber Kleinhenz (1994) für eine Diskussion). In jedem Fall würde seine Kategorie der phonologischen Phrase im Deutschen nicht in das Schema von Nespor/Vogel passen. 
(10)

[der alte Mann am Strand][fing heute] [besonders viele] [Fische] oder [besonders viele Fische]

Die Tatsache, daB die Intuitionen der Muttersprachler über die Phrasierung im Deutschen offenbar auseinandergehen, mag als erster Hinweis auf eine mangelnde Evidenz für die $\mathrm{PPh}$ gelten. Entscheidend ist aber der Mangel an phonologischen Regeln, die sich auf die Konstituente PPh beziehen. Diese Eigenschaft teilt das Deutsche mit anderen Sprachen (vgl. (2.2). In (11) sind einige dieser Sprachen aufgeführt.

(11) Phonologische Phrasen als Regeldomäne

Sprache

(a)

Französisch

Mandarin

Bengali

Italienisch

(b)

Englisch

Portugiesisch

Niederländisch

Deutsch
Beispiel

Liaison (Nespor/Vogel 1986)

Dritter Ton Sandhi (Cheng 1970)

r-Assimilierung (Hayes/Lahiri 1991)

RS (Nespor/Vogel 1986)

nur Akzenttilgung

keine (Frota 1995)

nur Akzenttilgung

nur Akzenttilgung (phon. Phrasen = synt.

Phrasen)

Nur die Sprachen in (11a) haben phonologische Regeln, die systematisch die Grenzen phonologischer Phrasen markieren, wie z.B. das Radoppiamento Sintattico im Italienischen (vgl. (5)). Die Sprachen unter (11b) haben zwar die rhythmisch bedingte Akzenttilgung (vgl. (3)), der entscheidende Unterschied zu den Sprachen in (11a) ist jedoch, dab die Domäne der Akzenttilgung immer auch eine syntaktische Domäne ist (z.B. Kopf und Modifizierer). Damit sind die angenommenen "phonologischen Phrasen" noch keine eigenen Domänen.

Die Aufstellung unter (11) erscheint zunächst wie eine ziemlich willkürliche Ansammlung von Sprachen, von denen einige offenbar dazu neigen, phonologische Phrasen als Regeldomäne zu verwenden.

Die Frage bleibt, wie man dies bewerten soll. Dafür sind zwei Möglichkeiten denkbar. Man kann (wie 2.B. Auer (1991, S. 105)) sagen, daB sich Sprachen hinsichtlich der Anzahl ihrer prosodischen Kategorien unterscheiden können. Solange davon aber keine weiteren Argumente abhängen, ist es im Hinblick auf die Theorie angemessener zu sagen, daß 
alle Sprachen über alle prosodischen Kategorien verfügen, daß aber in manchen Sprachen nicht alle "aktiv" sind (vgl. Frota 1995, S. 17). In diesem Beitrag soll untersucht werden, ob die Kategorie der phonologischen Phrase tatsächlich universell ist, oder ob die Existenz phonologischer Phrasen als Regeldomäne weitere Eigenschaften der betreffenden Sprachen impliziert.

Meine Hypothese ist, daß die Neigung von Sprachen, den Rändern von phonologischen Phrasen eine Funktion zuzuweisen, mit anderen Eigenschaften dieser Sprachen korreliert, und zwar genau mit den Eigenschaften, die andere Autoren für die Wortakzenttypologie aufgestellt haben.

1.2 Typologische Eigenschaften auf der Wortebene: silbenzählende vs. akzentzählende Sprachen

Auf der Wortebene gibt es zahlreiche Bemühungen um eine typologische Einteilung der einzelsprachlichen Eigenschaften von Rhythmus. In der Phonetik unterschied man schon früh zwei Sprachrhythmustypen, die silben- und die akzentzählenden Sprachen. Diese Unterscheidung geht auf Pike (1945) zurück. In silbenzählenden Sprachen sollte demnach die Dauer der einzelnen Silben immer konstant sein, in akzentzählenden Sprachen dagegen der Abstand zwischen den betonten Silben.

Aus diesen unterschiedlichen rhythmischen Grundbestrebungen der Sprachen ergeben sich weitere Eigenschaften, die eine Einteilung in diese zwei Sprachtypen rechtfertigten. So neigen nur akzentzählende Sprachen dazu, Silben in unbetonter Stellung zu reduzieren, um so die Abstände zwischen den Akzenten gleich zu halten. Silbenzählende Sprachen dagegen tendieren dazu, ihre Silben zu vereinheitlichen. Sie unterliegen typischerweise phonologischen Prozessen, die in einem CV-Silbenschema resultieren.

Mit der Weiterentwicklung und Verfeinerung phonetischer Meßmethoden hat sich allerdings herausgestellt, daß diese Unterscheidung so nicht zu halten ist. ${ }^{4}$ In einer eher phonologisch basierten Arbeit schlägt Auer (1991) eine alternative Rhythmustypologie vor, wobei er weitere typologische Parameter hinzuzieht. Auer gelangt so zu einer Einteilung in silbenbetonte und wortbetonte Sprachen. Dabei verwendet er im wesentlichen die Einteilungskriterien, die schon aus den phonetischen $\mathrm{Ar}$ beiten bekannt sind, allerdings mit zwei wichtigen Unterschieden: Zum einen betont er, daß eine binäre Aufteilung aller Sprachen in diese Typen nicht möglich ist. Statt dessen sieht er einen graduellen Übergang vor

4 Für einen Ūberblick über die Kritik vgl. Auer (1991); Mooshammer (1994). 
(vgl. (13)). ${ }^{5}$ Außerdem hat Auer eine funktionale Erklärung für die Eigenschaften, die jeweils mit den Typen "silbenbetont" und "wortbetont" korrelieren. In Sprachen vom letzteren Typ (wie z.B. dem Englischen) ist das Wort die wichtigste Domäne, da sich diese Sprachen rhythmisch am Wortakzent orientieren. Wortgrenzen fallen in diesen Sprachen immer mit Silbengrenzen zusammen und sind daher einfach zu erkennen. Daraus resultieren die anderen Eigenschaften dieses Sprachtyps. Sprachen vom silbenbetonten Typ (wie z.B. das Französische) dagegen haben typischerweise Sandhiregeln (die Wortgrenzen ignorieren) um das CV-Silbenschema zu erfüllen. Diese Sprachen müssen ihre Wortgrenzen auf andere Weise markieren, nämlich durch segmentale Regeln, die sich zumeist auf Silben beziehen. Die Liste in (12) basiert auf Auers Einteilungen.

(12) Sprachrhythmustypologie (nach Auer 1991)

$\begin{array}{ll}\begin{array}{l}\text { Rhythmus silbenbetont } \\ \text { eher Tonsprache }\end{array} & \begin{array}{l}\text { Rhythmus wortbetont } \\ \text { eher keine Tonsprache } \\ \text { keine reduzierten Vokale }\end{array} \\ \begin{array}{l}\text { unbetonte Vokale werden reduziert } \\ \text { einfache Silben }\end{array} & \begin{array}{l}\text { komplexe Silben } \\ \text { keine Liaison }\end{array} \\ \text { Liaison } & \begin{array}{l}\text { Geminaten nicht möglich } \\ \text { Geminaten möglich }\end{array} \\ \text { keine wortbezogenen Regeln } & \text { wortbezogene Regeln } \\ \text { keine silbenbezogenen Regeln } & \text { silbenbezogene Regeln }\end{array}$

Anhand dieser (und ähnlicher) Kriterien hat Auer ein Korpus von 34 Sprachen auf einer Skala zwischen dem Silbentyp und dem Worttyp eingeteilt.

(13) Kontinuum vom „Silbenpol” zum „Wortpol” (nach Auer 1991)

Silbensprachen .......................Wortsprachen

$\begin{array}{lll}\text { Yoruba } & \text { Mandarin Französisch Uzbekisch } & \text { Englisch } \\ \text { Vietnamesisch } & \text { Japanisch Koreanisch } & \text { Russisch } \\ & \text { Hausa } & \text { Arabisch }\end{array}$

An Auers Vorschlag sind meiner Meinung nach einige Punkte problematisch. Sie betreffen die Behauptung, daB dem "Wortrhythmustyp" ein "Silbenrhythmustyp" entgegengesetzt ist, und daB sich nur der letztere regelhaft auf die Silbe beziehen kann. Es erscheint mir wenig sinnvoll,

5 In diese Richtung geht auch Nespor (1990), die gegen eine Parametrisierung von Sprachrhythmuseigenschaften in der Phonologie argumentiert. 
Sprachen anhand ihrer Silbenbezogenheit zu unterscheiden. Die Silbe ist eine Regeldomäne, auf die alle Sprachen in mehr oder weniger starkem Maße zurückgreifen. Die Kriterien von Auer, die sich auf Silbengrenzen beziehen, lassen sich nicht einmal auf alle Sprachen in seinem Korpus anwenden (so hat z.B. das Arabische Geminaten, obwohl es bei Auer dem extremen Gegenpol zum Silbenrhythmus zugeordnet ist). Eine andere Sprache, die man dem Wortrhythmustyp zuordnen würde, ist das Deutsche (es fehlt in Auers Korpus). Im Deutschen gibt es jedoch gleichzeitig eine Vielzahl von silbenbezogenen Regeln (vgl. Hall 1992) ${ }^{6}$

Im Gegensatz dazu ist Auers funktionale Motivation für den Sprachtyp "Wortrhythmus" überzeugend: Wortgrenzen fallen in diesen Sprachen immer mit Silbengrenzen zusammen. Regeln, die über Wortgrenzen hinausgehen, kommen (im Gegensatz zu dem Silbentyp, vgl. die französische Liaison in (14)) hier nicht vor. Daher ist zu erwarten, daß in den Worttypsprachen dem Wortende eine größere phonologische Bedeutung - im Sinne von Regeldomänen - zugewiesen wird.

Meiner Meinung nach ist jedoch der Gegenpol zu diesem Sprachtyp nicht ein Sprachtyp, der eine kleinere Regeldomäne (wie die Silbe) bevorzugt, sondern einer, der die phonologische Phrase als Domäne verwendet.

\subsection{Phrasenebene: typologische Korrelate}

In diesem Abschnitt möchte ich die Vermutung aufstellen, daß es eine Korrelation gibt zwischen dem Trend einer Sprache zum Silbentyp nach Auer und dem Trend, phonologische Phrasen mit Funktionen zu beladen. Hierzu vergleiche ich Sprachen vom Worttyp und vom Silbentyp.

In Sprachen vom Worttyp ist die Wortgrenze zugleich Barriere für phonologische Regeln, mit anderen Worten, Wortgrenzen werden markiert, indem phonologische Regeln an dieser Stelle unterbrochen werden. Weitere Mittel zur Markierung von Grenzen stehen in diesen Sprachen nicht zur Verfügung, weil unbetonte Silben zugleich reduziert werden, d.h., akustisch sind die Grenzen oft nicht wahrnehmbar. Aufgrund der Akzentverhältnisse wäre es in solchen Sprachen schwer, ein Phrasenende zu

6 Auer würde dieser Auffassung nicht zustimmen: Er hat argumentiert (z.B. in Auer (1994)), daß die Silbe keine Hauptkategorie im Deutschen ist, indem er zeigt, daß man die Auslautverhärtung ohne Bezug auf die Kategorie Silbe erklären kann. Er diskutiert aber nicht die kritischen Beispiele wie re/k/nen (Hall, persönliche Mitteilung). Ich möchte die Diskussion, ob die Silbe tatsächlich eine universale Kategorie ist, hier nicht vertiefen. Wichtig ist mir in diesem Zusammenhang, daß bei der Einteilung in „Wort-” und "Silbensprachen" die Kriterien, die auf der Einheit Silbe beruhen, nicht so nachvollziehbar sind wie die, die auf der Einheit Wort beruhen. 
markieren: falls der lexikalische Akzent nicht zufälig auf die letzte Silbe fällt, kann er nicht dorthin bewegt werden. Aufgrund dieser fehlenden Prominenz könnte die phrasenfinale Silbe nur schwer dazu dienen, in der Phonologie etwas zu markieren.

Wenn man annimmt, daB alle Sprachen in der einen oder anderen Form dem "Silbentyp” angehören, so stellt sich die Frage, welche Strategie die Sprachen verwenden, die nicht unter den Wortrhythmustyp fallen, also etwa Französisch, Italienisch, viele Tonsprachen. Das phonologische Wort kann in diesen Sprachen nur schwer als Regeldomäne dienen: Dessen Grenzen verschwimmen häufig zugunsten der Silbe. Charakteristisch für diese Sprachen sind Resilbifizierungen über Wortgrenzen hinweg, wie die französische Liaison. Als Resultat dieser Liaison entstehen größere artikulatorische Einheiten - vgl. (14). Daraus kann sich dann eine Tendenz in der Phonologie entwickeln, die Grenzen dieser Einheiten mit einer Funktion zu versehen. Das heißt, es wäre zu erwarten, daß Sprachen, die keine ausgeprägten phonologischen Wörter haben, eher dazu neigen, phonologische Phrasen als Regeldomäne zu entwickeln, als Sprachen, bei denen die entsprechenden Regeln bereits auf der Wortebene lokalisiert sind. Demzufolge könnten Sprachen solche phonologischen Phrasen als eigene Domäne entwickeln. In einem solchen Entwicklungsproze $B$ verwandeln sich die ursprünglichen Phrasen, die mit den syntaktischen Phrasen übereinstimmen und (wie in 1.1 beschrieben) die Domäne von Phrasenakzenten sind, in unabhängige Domänen. Dadurch sind sie nicht mehr isomorph mit syntaktischen Phrasen. Vermutlich können Sprachen solche Domänen ausbilden oder verlieren. Im nächsten Abschnitt illustriere ich dies anhand der romanischen Sprachen Italienisch, Französisch und Portugiesisch.

Danach zeige ich, wie diese Idee zu einer Sprachrhythmustypologie beitragen könnte.

\section{Beispiele}

In diesem Abschnitt soll die oben aufgestellte Hypothese anhand von Sprachen, die unterschiedlichen Sprachrhythmustypen entsprechen, überprüft werden. Die Frage lautet, ob man wirklich den „Silbentyp” zugunsten eines "Phrasentyps” abschaffen kann. Mit anderen Worten: Korrelieren Sprachen, die dem "Silbentyp" entsprechen, mit den Sprachen, die eigene phonologische Phrasen haben? 


\title{
2.1 Italienisch - Französisch - Portugiesisch
}

Laut Auer entspricht Italienisch (wie auch Latein) dem Prototyp einer Silbentypsprache (so hat es Geminaten, keine reduzierten Vokale, einfache Silben); Französisch hat laut Auer eine "Tendenz" zum Silbentyp, es weist aber weniger Eigenschaften dieses Typs auf und läßt sich daher nicht so gut zuordnen.

Das Portugiesische wurde von Auer nicht untersucht. Es bietet sich hier als Vergleich an, weil es sich nach Auers Kriterien zu einer Worttypsprache entwickelt haben müBte: Es hat keine Geminaten, alle unbetonten Vokale werden $\mathrm{zu}[u]$ reduziert und es hat komplexe Silben.

Die Frage lautet nun, wie sich diese Sprachen hinsichtlich größerer phonologischer Domänen verhalten. Im Italienischen sind phonologische Phrasen eigene Domänen (z.B. Radoppiamento Sintattico (6)). Im Französischen gibt es eine Resilbifizierungsregel, die über Wortgrenzen hinweg operiert, aber von Phrasengrenzen blockiert wird, die Liaison. Silbenfinale Konsonanten werden im Französischen nicht realisiert. Durch Liaison können sie zum Onset einer folgenden vokalinitialen Silbe werden. Wie (14) zeigt, operiert diese Regel aber nur innerhalb der Domäne der phonologischen Phrase, also [les enfants] [sont allés] etc., nicht zwischen diesen Domänen, auch wenn die segmentale Umgebung dafür gegeben wäre.

(14) Französische Liaison (vgl. Nespor/Vogel 1986)

\author{
les enfants sont allés à l'école \\ die Kinder sind gegangen in die Schule \\ [le:.zãfã.s5.ta.le: ?a] \\ aber nicht \\ [s כ̃.ta.le.za]
}

Im Portugiesischen gibt es überhaupt keine Evidenz für phonologische Phrasen, wie Frota (1995) gezeigt hat. Sie hat alle Regeln, die über Wortgrenzen hinweg operieren, untersucht und gezeigt, daß deren Domäne nicht die phonologische Phrase sein kann.

Diesen Beobachtungen zufolge korreliert also die "Silbentypsprache” Italienisch mit dem von mir hier postulierten „Phrasentyp”, die „Worttypsprache" Portugiesisch hat dagegen keine phonologischen Phrasen. Das Französische läßt sich mit dem neu hinzugekommenen Kriterium der Phrase nun deutlicher mit dem Italienischen zusammen gruppieren. In Abschnitt 3 erläutere ich den „Phrasentyp” ausführlicher. 


\subsection{In welche Kategorie gehört das Deutsche?}

Wie schon gesagt ist das Deutsche ein Beispiel für eine Sprache, die sich nicht einem von Auers Rhythmustypen zuweisen läBt. Es läßt sich aber nicht in die Mitte zwischen den Polen einordnen, weil es einerseits die Eigenschaften von wortbetonten Sprachen aufweist, andererseits aber viele silbenbezogene Regeln hat (vgl. Hall 1992). Es entspricht also beiden Typen. Meiner Meinung nach ist dies darauf zurückzuführen, dab die Silbe eine universelle Konstituente ist, deren Grenzen in allen Sprachen phonologisch relevant sind.

Wie läßt sich das Deutsche anhand einer Einteilung in Wort- und Phrasensprachen klassifizieren? Wenn man Auers Typologie hinsichtlich der Eigenschaften von Wortsprachen beibehält, ist das Deutsche klar eine Wortsprache. Das sagt auch Auer (1994). Allerdings muB er dann den Stellenwert der silbenbezogenen Regeln im Deutschen typologisch neu bewerten.

Eine Einteilung in wort- vs. phrasenbezogene Sprachen würde die Vorliersage machen, daß das Deutsche keine phonologischen Phrasen hat.

Nun hat das Deutsche aber eine Regel, die möglicherweise auf phonologischen Phrasen operiert, die "Rhythm Rule” (vgl. auch (3) oben).

(15) Akzentabschwächung (Rhythm Rule) im Deutschen

$\begin{array}{lll}\text { KONstanz } & \text { aber } & \text { Konstanzer ÚNi } \\ & \text { aber } & \text { [die ÚNi] [von KONstanz] }\end{array}$

Allerdings genügt dies allein noch nicht, um von einer unabhängigen $\mathrm{Ka}$ tegorie "phonologische Phrase” zu sprechen. Erstens ergeben sich Akzentuierung und Akzentreduktion direkt aus der syntaktischen Beziehung der Konstituenten.

Außerdem ist die Akzentuierung wie auch die Akzentverschiebung Teil der Prosodie, die einen anderen Stellenwert in der Grammatik hat als die eigentlichen phonologischen Regeln, auch die sog. "postlexikalischen” Regeln, d.h. phonologische Regeln, die erst nach dem Lexikon applizieren. In dem Modell von Selkirk (16), welches hinsichtlich der Rolle der Prosodie in der Grammatik als Standardmodell angesehen werden kann, sieht man, daß sich die Prosodie direkt auf die Syntax beziehen kann. Erst anschließend an den AkzentuierungsprozeB erfolgt die postzyklische Phonologie. Auf diese Regeln aber beziehen sich die Domänen der Phonologie (z.B. die französische Liaison, das Radoppiamento Sintattico im Italienischen). 
(16) Prosodie und Deakzentuierung in der Grammatik (nach Selkirk 1984, S. 34)

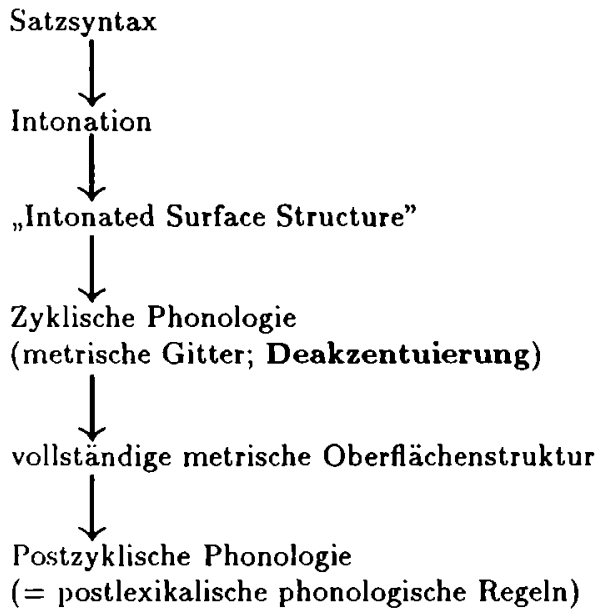

In der Literatur wird oft kein Unterschied zwischen Prosodie und postlexikalischer Phonologie gemacht, weil er normalerweise nicht relevant ist. Wenn man aber, wie im Rahmen der vorliegenden Problemstellung, $\mathbf{z w i}$ schen „Wort-” und „Phrasensprachen” unterscheiden will, so wird dieser Unterschied wichtig. Man findet in der Phonologie des Deutschen keine Evidenz für phonologische Konstituenten in Phrasengröße im eigentlichen Sinne, d.h. als von der Syntax abweichende Domänen postzyklischer Regeln (vgl. die Kriterien in (1)). Eine Regel, die sich auf das syntaktische Verhältnis zwischen den Konstituenten bezieht, wie die Akzentabschwächung, kann nicht als Evidenz für eine unabhängige phonologische Domäne gelten.

\section{Zusammenfassung: wort- vs. phrasenbezogene Sprachen}

In wortbetonten Sprachen spielen Phrasengrenzen als Regeldomäne keine Rolle. Phonologische Phrasen, wenn es sie überhaupt gibt, fallen stets mit syntaktischen Phrasen zusammen und erfüllen damit nicht das Kriterium einer unabhängigen Domäne gemäß (1). Andererseits ist aber die Silbe auch in vielen wortbetonten Sprachen (närnlich wohl allen NichtTonsprachen) eine wichtige Konstituente.

Ich habe daher für eine andere Unterscheidung argumentiert, nämlich für eine Differenzierung in wort-und phrasenbezogene Sprachen. Für diese 
Unterscheidung kann man viele von Auers Beobachtungen hinsichtlich der Worttypsprachen beibehalten, einige silbenbezogene Regularitäten allerdings nicht.

Umgekehrt möchte ich dem typologischen Raster ein wichtiges Einteilungskriterium hinzufügen, nämlich die Isomorphie mit morphosyntaktischen Einheiten.

(17) Erweiterung von Auers Typologie auf Phrasen

phrasenbezogen

eher Tonsprache

keine reduzierten Vokale

Liaison

phonologisches Wort $=$ morpho-

syntaktisches Wort

phonologische Phrase $\neq$ syn-

taktische Phrase

\section{wortbezogen}

eher keine Tonsprache

unbetonte Vokale werden

reduziert

keine Liaison

phonologisches Wort $\neq$ morphosyntaktisches Wort

wenn phonologische Phrase, dann $=$ syntaktische Phrase

Phrasenbezogene Sprachen haben der Domäne der Phrase und ihren Grenzen ein großes funktionales Gewicht verliehen. Daher sind die Wortgrenzen weniger relevant, sie können innerhalb der Phrase verschwimmen (z.B. Französisch bei der Liaison). In wortbetonten Sprachen (wie dem Deutschen) übernehmen die Wortgrenzen viele Funktionen der Phrasengrenzen. Daher können sie nicht noch mit weiteren Funktionen befrachtet werden. Die Silbe steht beiden Sprachtypen zur Verfügung, wird aber erwartungsgemäß von den phrasenbetonten Sprachen stärker genutzt, da viele Regeltypen kleine Domänen, wie Silbe oder Wort erfordern (z.B. lokale Assimilationen).

Es ist nicht sinnvoll, anzunehmen, daß Sprachen, die keine overte Markierung von phonologischen Phrasengrenzen haben, diese „unsichtbar” doch haben, weil man dann eine typologische Generalisierung verlieren würde. Allerdings können Sprachen phonologische Phrasen entwickeln. In der ersten Stufe werden syntaktische Phrasen verwendet, um phonologische Domänen zu markieren, wie die Akzentreduzierung im Deutschen oder Englischen. In der nächsten Stufe entwickeln diese Phrasen eigene GesetzmäBigkeiten, so daß sie nicht mehr mit den syntaktischen Phrasen zusammenfallen. Dies erfüllt dann ein Charakteristikum einer phrasenbetonten Sprache. Unabhängig von diesem graduellen Übergang ist aber anhand des Vorhandenseins unabhängiger phonologischer Phrasen als Regeldomäne eine eindeutige Klassifizierung der Sprachen möglich. 
Im Schema ist dieser graduelle Übergang dargestellt:

Wortsprachen

Deutsch

Portugiesisch
Phrasensprachen

Französisch Italienisch

\section{Literatur}

Auer, Peter (1991): Is a Rhythm-Based Typology Possible? A Study on the Role of Prosody in Phonological Typology. Universitāt Konstanz: KontRI Working Papers 21.

Auer, Peter (1994): Einige Argumente gegen die Silbe als universale prosodische Hauptkategorie. In: Ramers, Karl-Heinz et al. (Hg.): Universale phonologische Strukturen und Prozesse. Tübingen. S. 55-78.

Cheng, Chin-Chuan (1970): Domains of Phonological Rule Application. In: Saddock, J.M./Vanek, A.L. (Hg.): Studies presented to Robert B. Lees by his students. Edmonton: Linguistic Research, S. 39-59.

Frota, Sonia (1995): Prosodic Phrases and European Portuguese: in Search of Evidence. Erscheint in: Proceedings of ConSole III, Venedig.

Giegerich, Heinz (1985): Metrical Phonology and Phonological Structure. German and English. Cambridge.

Hall, T.A. (1992): Syllable Structure and Syllable-Related Processes in German. Tübingen.

Hayes, Bruce/Lahiri, Aditi (1991): Bengali Intonational Phonology. In: Natural Language and Linguistic Theory 9, S. 47-96.

Kleinhenz, Ursula (1994): Focus and Phrasing in German. Stuttgart/Tübingen: Arbeitspapiere des Sonderforschungsbereichs 340. Nr. 52.

Mooshammer, Christine (1994): Sprachrhy thmustypologie. Akzentzählende vs. silbenzählende Sprachen. MS. Berlin/München.

Nespor, Marina/Vogel, Irene (1986): Prosodic Phonology. Dordrecht.

Nespor, Marina (1990): On the Rhythm Parameter in Phonology. In: Roca, I. (Hg.): Logical Issues in Language Acquisition. Dordrecht. S. 157-173.

Pike, Kenneth (1945): The Intonation of American English. Ann Arbor.

Rice, Keren D. (1990): Predicting Rule Domains in the Phrasal Phonology. In: Inkelas, Sharon/Zec, D. (Hg.): The Phonology-Syntax Connection. Chicago. S. 289-312.

Selkirk, Elizabeth (1980): Prosodic Domains in Phonology: Sanskrit Revisited. In: Aronoff, Mark/Kean, M.L. (Hg.): Juncture. Saratoga. S. 107-129.

Selkirk, Elizabeth (1984): Phonology and Syntax: The Relation between Sound and Structure. Cambridge, Mass.

Wiese, Richard (1995): The Phonology of German. Oxford. 